\title{
Modelização do Programa Nacional de Melhoria do Acesso e da Qualidade em um município baiano
}

\author{
Modelling of the National Program for Improving Primary Care \\ Access and Quality in a municipality from Bahia
}

Larissa Morgan Andrade Lemos', Nília Maria de Brito Lima Prado', Maria Guadalupe Medina'

DOI: 10.1590/0103-1104202012501

RESUMO O Programa Nacional de Melhoria do Acesso e da Qualidade da Atenção Básica é uma iniciativa importante para o fortalecimento da Atenção Primária à Saúde no País, na perspectiva de melhoria e mudança do modelo de atenção à saúde em consonância com os princípios orientadores da Reforma Sanitária Brasileira. Este artigo objetiva analisar e explicitar, por meio de um modelo lógico-operacional referente aos dois primeiros ciclos, a plausibilidade lógica do Programa em um município baiano. Para tanto, foi realizado um estudo avaliativo de caso único no município investigado. A produção de dados foi realizada mediante entrevistas semiestruturadas, que objetivaram extrair dos gestores da atenção básica e profissionais de duas Unidades de Saúde da Família os relatos sobre suas experiências com a implementação de ações vinculadas ao Programa. Foi possível descrever o contexto de sua implementação no município, identificar seus objetivos, as ações desenvolvidas pelas equipes e a percepção dos atores quanto aos principais resultados alcançados no município, bem como verificar a coerência, no nível local, em comparação com as recomendações nacionais, propondo um modelo de análise útil para os gestores do Programa.

PALAVRAS-CHAVE Atenção Primária à Saúde. Política de saúde. Avaliação em saúde. Avaliação da qualidade dos cuidados de saúde. Programas nacionais de saúde.

ABSTRACT The National Program for Improving Primary Care Access and Quality (PMAQ-AB) is an important initiative to strengthen Primary Health Care in the Country, aiming at improving and changing the health care model in line with the guiding principles of the Brazilian Sanitary Reform. This article aims to analyze and explain, through an operational logical model referring to the first two cycles, the logical plausibility of the Program in a municipality in Bahia. For that, a single case evaluative study was carried out in the municipality under investigation. The data were produced through semi-structured interviews, which aimed to extract from the primary care managers and professionals of two Family Health units the reports about their experiences with the implementation of actions linked to the Program. It was possible to describe the context of its implementation in the municipality, to identify its objectives, the actions developed by the teams and the main results achieved in the municipality, as well as to verify the coherence, at the local level in comparison with the national recommendations, proposing a useful analytical model for program managers.

1 Universidade Federal da Bahia (UFBA) - Vitória da Conquista (BA), Brasil.

larissamorgan@fainor.com.br
KEYWORDS Primary Health Care. Health policy. Health assessment. Evaluation of the quality of health care. National health programs. 


\section{Introdução}

O movimento da Reforma Sanitária Brasileira busca a reorientação do modelo de atenção à saúde, a melhoria da cobertura e da qualidade dos serviços, bem como a integralidade das práticas, aprofundando processos de democratização e de participação social1,2.

Para superar o modelo de saúde centrado na doença e em práticas curativas, a Estratégia Saúde da Família (ESF) tem sido priorizada como alternativa para o fortalecimento de um modelo de Atenção Primária à Saúde (APS) ${ }^{\mathbf{3}, \mathbf{4}}$. Contudo, apesar de essa estratégia ter promovido a qualificação e uma maior abrangência às ações de caráter interdisciplinar e interprofissional, do mesmo modo, desvelou alguns desafios para o Sistema Único de Saúde (SUS), relacionados à melhoria dos processos assistenciais, a condições para efetivar a integração da APS à rede de serviços de saúde em seus diversos níveis de atenção e à dificuldade na qualificação das equipes da Atenção Básica (AB) ${ }^{\mathbf{1}, 4}$.

Para reverter esse cenário, a aposta governamental mais recente é o Programa Nacional de Melhoria do Acesso e da Qualidade da Atenção Básica (PMAQ-AB) ${ }^{5}$, uma iniciativa destinada a induzir as capacidades dos gestores e das equipes de $\mathrm{AB}$ a ampliarem o acesso e a qualidade dos serviços de saúde ${ }^{6,7}$.

Contudo, ainda são incipientes os estudos sobre o PMAQ-AB que revelem as nuances, inconsistências e contradições do Programa. Ressalta-se, ainda, a existência de heterogeneidade na implementação local, especialmente relacionada à destinação dos incentivos financeiros, e a coerência e integridade do Programa em relação ao escopo idealizado e proposto pela normatização federal ${ }^{8-11}$. Diante disto, surge o questionamento sobre em que medida o desenho do PMAQ-AB é capaz de assegurar os efeitos pretendidos, especialmente em municípios com dificuldades para a sua implementação. Assim, o presente estudo teve como objetivo analisar e explicitar, por meio de um modelo lógico operacional referente aos dois primeiros ciclos, a plausibilidade lógica do PMAQ-AB em um município baiano.

\section{Material e métodos}

Foi realizado um estudo de caso único com o intuito de modelizar o PMAQ-AB implementado em um município de médio porte do estado da Bahia, no período compreendido entre agosto de 2017 e junho de 2018.

No planejamento de um processo avaliativo, a elaboração do modelo lógico configura-se como um dos passos iniciais. Este modelo pode ser definido como um esquema visual, que apresenta como uma intervenção deve ser implementada e quais resultados são esperados $^{12}$. A modelização revela também o conjunto de hipóteses necessárias para que a intervenção permita melhorar determinada situação problemática, sendo a sistematização destas hipóteses a teoria do Programa ${ }^{13}$.

A modelização favorece que uma iniciativa seja compreendida em sua racionalidade e em seu encadeamento lógico, abrangendo componentes tais como problemas, pressupostos, objetivos, hipóteses, estratégias e resultados esperados, explicitados em um modelo lógico-operacional13.

O caso selecionado para esta pesquisa foi um município de referência macrorregional da região Sudoeste do estado da Bahia, que apresenta uma ampla rede de atenção primária, organizada nas zonas urbana e rural por unidades básicas, Equipes de Saúde da Família (EqSF) e Núcleo Ampliado de Apoio à Saúde da Família (Nasf) ${ }^{\mathbf{1 4}}$.

Para aprofundar a análise sobre os fundamentos e a lógica da implementação do PMAQ-AB no município, foram selecionadas duas EqSF (E1 e E2) que obtiveram pontuação acima da média na avaliação de desempenho, nos dois primeiros ciclos do PMAQ-AB. A primeira equipe, vinculada a uma Unidade de Saúde da Família (USF) localizada na zona rural (USF 1), e a segunda equipe, vinculada a uma USF da zona urbana (USF 2).

As entrevistas foram realizadas com gestores da atenção primária e profissionais das duas EqSF que haviam trabalhado no município nos dois primeiros 
ciclos do PMAQ-AB. Quanto aos Agentes Comunitários de Saúde (ACS), foram incluídos aqueles com o maior tempo de atividade profissional na unidade. Assim, foram realizadas 11 entrevistas, com 2 médicos, 2 enfermeiros, 4 ACS e 3 gestores (quadro 1).

Todas as entrevistas foram previamente agendadas e realizadas pelo pesquisador principal, em espaços distintos das unidades de saúde, para evitar constrangimentos decorrentes de algum questionamento relativo às práticas profissionais. Antes de se iniciarem as entrevistas, o consentimento informado por escrito foi obtido, sendo assegurados aos profissionais o sigilo e a confidencialidade das informações.

Os dados derivados das entrevistas foram transcritos na íntegra e organizados de modo a permitir a identificação de convergências e divergências nos discursos dos entrevistados, sendo realizada a triangulação das fontes de evidência, mediante entrevistas transcritas e observações in loco realizadas pelo pesquisador.

A análise dos dados contemplou as seguintes categorias: (1) objetivos do Programa; (2) ações e/ou atividades desenvolvidas; (3) processo de implantação (reorganização da atenção, processo de trabalho, infraestrutura); (4) remuneração (incentivo individual, investimentos em infraestrutura); (5) avaliação (indicadores, avaliação externa); (6) resultados; (7) percepção sobre o PMAQ-AB. A análise com base nas categorias operacionais permitiu elucidar o modelo lógico-operacional do PMAQ-AB no município, bem como verificar a coerência do Programa em comparação com a proposta governamental. Após a elaboração do modelo lógico- operacional local, foi realizada uma comparação com o modelo teórico do PMAQ-AB proposto em um estudo de avaliação ${ }^{15}$.

Quadro 1. Caracterização dos participantes da pesquisa, de acordo com escolaridade e tempo de atuação na APS ou gestão

\begin{tabular}{lllll}
\hline Vínculo institucional & Cargo ocupado & $\begin{array}{l}\text { Código do } \\
\text { entrevistado }\end{array}$ & Escolaridade & $\begin{array}{l}\text { Tempo de atuação na } \\
\text { APS/Gestão }\end{array}$ \\
\hline Equipes de saúde & Enfermeiro & E1U1 & E. Superior & 15 anos \\
& Médico & E2U1 & E. Superior & anos \\
& $\begin{array}{l}\text { Agente Comunitário } \\
\text { de Saúde }\end{array}$ & E3U1 & E. Médio & 19 anos \\
& $\begin{array}{l}\text { Agente Comunitário } \\
\text { de Saúde }\end{array}$ & E4U1 & E. Fundamental & 18 anos \\
& $\begin{array}{l}\text { Enfermeiro } \\
\text { Médico }\end{array}$ & E5U2 & E. Superior & 15 anos \\
& $\begin{array}{l}\text { Agente Comunitário } \\
\text { de Saúde }\end{array}$ & E7U2 & E. Superior & 20 anos \\
& $\begin{array}{l}\text { Agente Comunitário } \\
\text { de Saúde }\end{array}$ & E8U2 & E. Médio & 15 anos \\
Gestor da APS & E9G & E. Superior & 17 anos \\
Gestor da APS & E10G & E. Superior & 3 anos \\
Gestor da SMS* & E11G & E. Superior & 12 anos \\
Gestão & & E. Superior & 5 anos \\
\hline
\end{tabular}

Fonte: Elaboração própria.

*SMS: Secretaria Municipal de Saúde. 
A análise de coerência contemplou as seguintes fases: 1. Explicação do problema e referências básicas do Programa; 2. Estruturação lógica do Programa para alcance de resultados (objetivos, ações, resultados); 3. Identificação de fatores de contexto que podem influenciar na implementação do Programa; 4. Comparação entre o modelo lógico-operacional do Programa e o modelo teórico elaborado a partir da revisão da literatura ${ }^{16}$.

O projeto foi aprovado pelo Comitê de Ética em Saúde da Universidade Federal da Bahia, do Instituto Multidisciplinar em Saúde, sob o parecer $\mathrm{n}^{\circ} 2.475 .022$, e seguiu as normas regulamentadoras de pesquisas envolvendo seres humanos.

\section{Resultados}

\section{$O$ contexto anterior à implementação do PMAQ-AB no município}

O município investigado foi um dos pioneiros na região Nordeste a implantar a ESF. Iniciou com a implantação do Programa de Agentes Comunitários de Saúde, ocorrendo a primeira seleção de agentes em abril de 1998. Em setembro do mesmo ano, implantou o Programa de Saúde da Família (PSF) com cinco equipes na área urbana, cada uma contando com um médico, um enfermeiro, um odontólogo, três auxiliares de enfermagem e quatro ACS. Já no ano de 2001, o PSF alcançou 30 equipes de saúde e uma cobertura de $40,03 \%$. Em 2010, essa cobertura foi ampliada para $41,11 \%$, contando com 38 equipes de saúde. Além da ESF, o município conta, na APS, com uma rede de serviços básicos que estão organizados com uma lógica de articulação entre as Unidades Básicas de Saúde (UBS), o Nasf e as USF, que são referências na organização dos fluxos para os demais níveis de complexidade da rede municipal.

O município aderiu ao PMAQ-AB desde o primeiro ciclo, em 2011, quando incluiu $31,5 \%$ de suas equipes, sendo que as razões mencionadas pelos gestores para essa adesão foram a inclusão de indicadores para a avaliação e o monitoramento das ações e serviços, e a ampliação da cobertura e dos recursos financeiros destinados a AB. Já os profissionais assinalaram que a adequação da estrutura física das USF incluídas e a ampliação dos recursos financeiros destinados à $\mathrm{AB}$ foram os principais fatores que motivaram a adesão.

Ao que parece, os gestores se empenharam em formalizar a adesão ao Programa por compreenderem a importância da avaliação no contexto da $\mathrm{AB}$, fato que deriva de suas experiências prévias de atuação neste âmbito, no próprio município investigado, antes de assumirem os cargos vinculados à gestão da $\mathrm{AB}$ e à secretaria municipal de saúde.

No que concerne a aspectos relacionados à gestão local e à organização dos serviços na $\mathrm{AB}$, o financiamento insuficiente para expandir e/ ou manter a cobertura dos serviços de saúde, ou ampliar ações de manutenção predial ou reestruturação física das unidades de saúde consideradas em condições inadequadas, foi a questão priorizada pelos gestores e demais profissionais. As fragilidades citadas pelos gestores apontaram, do mesmo modo, restrições relacionadas ao financiamento das ações para a ampliação da capacidade resolutiva da AB municipal. Um dos gestores pontuou a estagnação do valor dos repasses financeiros realizados pela esfera federal.

Além dos desafios supracitados, os gestores acrescentaram uma preocupação com a elevada rotatividade e, até mesmo, a ausência de profissionais de saúde nas USF - principalmente, os médicos -, na composição de algumas equipes. Para eles, tal fato poderia influenciar o processo de cuidado e a resolutividade dos problemas de saúde da população adstrita.

A rotatividade dos profissionais de saúde é um problema identificado no processo de implantação da ESF em diversos municípios brasileiros, tendo em vista que o modelo de atenção prioriza o vínculo entre profissionais da equipe de saúde e a população. A alta rotatividade pode estar relacionada à precarização 
dos vínculos empregatícios (poucos profissionais concursados atuando na $\mathrm{AB})^{\mathbf{1 7}}$.

Ainda sobre o contexto da implantação, alguns gestores apontaram potencialidades inerentes à $\mathrm{AB}$ do município investigado, no período antecedente à implementação do PMAQ-AB. Entre elas, pode-se citar a utilização de uma estratégia de avaliação denominada 'sala de situação', caracterizada pelo encontro periódico de representante de equipes de saúde com os apoiadores institucionais para a explicitação dos indicadores de saúde da unidade. Essa prática foi pontuada, de forma unânime entre profissionais e gestores, como uma potencialidade para a melhoria da qualidade técnica em saúde, pois demonstrava que a AB local incluía o monitoramento das ações e dos serviços como uma prática contínua.

A Sala de Situação em Saúde (SDSS) é uma ferramenta que favorece o uso da informação em saúde para a tomada de decisões, posto que seja uma proposta de trabalho que facilita a tarefa de analisar a informação sanitária e vinculá-la à gestão de governo em saúde. Assim, a SDSS apoia processos decisórios em duas direções: auxilia os gestores (municipais, estaduais ou federal) no seguimento ou na avaliação de políticas e programas de saúde; e subsidia a decisão que a equipe da sala realiza e que culmina nas UBS, onde se gera o dado primário ${ }^{\mathbf{1 8}}$.

\section{Modelo lógico-operacional do PMAQ-AB no município}

É importante destacar que a pertinência de uma intervenção depende, fundamentalmente, do contexto, dos atores envolvidos e do alinhamento entre objetivos e interesses, nos quais estão mergulhadas as intervenções em análise ${ }^{16}$. Neste sentido, a avaliação da intervenção pode contribuir para identificar se os objetivos definidos inicialmente são adequados para resolver o problema e responder as necessidades locais, e se os responsáveis pela intervenção estão agindo adequadamente, considerando suas funções e atribuições.
A análise prévia do contexto anterior à implementação do PMAQ-AB desvelou elementos importantes, quais sejam: financiamento insuficiente para a manutenção dos serviços da $\mathrm{AB}$, baixa cobertura da APS no município, unidades de saúde com estrutura inadequada, dificuldades para a expansão do número de equipes de saúde no município, realização de práticas de avaliação restritas à 'sala de situação' e equipes de saúde desestimuladas.

\section{$O$ contexto de implementação do PMAQ-AB no município}

Em 2011, o município aderiu ao primeiro ciclo do PMAQ-AB e, em 2013, sancionou a Lei Municipal $n^{\circ} 1.907$, que dispõe sobre o pagamento do incentivo financeiro do Programa. Por meio dela, a gestão estabeleceu que, dos recursos financeiros disponibilizados pelo PMAQ-AB, 50\% seriam utilizados para melhorar a adequação da estrutura da $A B$ municipal, não necessariamente nas unidades bem avaliadas. A segunda metade do recurso seria repassada, semestralmente, sob a forma de prêmio de incentivo, aos servidores lotados nas EqSF que aderiram ao Programa e à diretoria da $\mathrm{AB}$, condicionada ao desempenho de cada equipe, independentemente da categoria profissional e do montante de valores efetivamente recebidos pelo município por meio do Fundo Nacional de Saúde ${ }^{19}$.

No primeiro ciclo, 12 equipes dentre as 38 da ESF aderiram e participaram do PMAQ-AB. Dessas equipes, cinco obtiveram desempenho classificado como 'ótimo' e receberam $\mathrm{R} \$ 11$ mil por mês, que foram divididos entre o pagamento da premiação e o investimento na $\mathrm{AB}$, e sete equipes foram avaliadas com o conceito 'bom' e receberam um recurso correspondente a $\mathrm{R} \$ 6,6$ mil por mês para investir nas mesmas aplicações ${ }^{20}$.

No segundo ciclo, iniciado em 2013, o PMAQ-AB foi ampliado em todo o País, apresentando $74 \%$ de aumento no número 
de EqSF participantes, o que evidencia um grande incremento da adesão municipal. Entretanto, é importante destacar que, no primeiro ciclo, houve um limite previamente definido de equipes participantes, diferentemente do segundo, ao qual puderam aderir todas que desejaram ${ }^{\mathbf{2 1}}$.

No município investigado, 37 equipes de $A B$ aderiram ao segundo ciclo, o que representa $90 \%$ do total de equipes. Dessas, 16 obtiveram classificação 'muito acima da média', 19 foram avaliadas como 'acima da média' e 2 apresentaram avaliação 'mediana ou abaixo da média'20.

\section{Modelização do Programa Nacional de Melhoria do Acesso e da Qualidade da Atenção Básica no município}

A partir do reconhecimento do contexto da implantação, foi possível identificar os objetivos do Programa no município, as ações realizadas nesse território e os resultados alcançados em curto prazo, além de elaborar o modelo lógico-operacional, como se vê na figura 1, que faz uma análise dos relato dos atores sociais envolvidos na implementação do PMAQ-AB no município e verifica se condizem com as recomendações nacionais.

Figura 1. Modelo lógico-operacional do Programa Nacional de Melhoria do Acesso e da Qualidade da Atenção Básica em um município do sudoeste da Bahia

Contexto da implantação

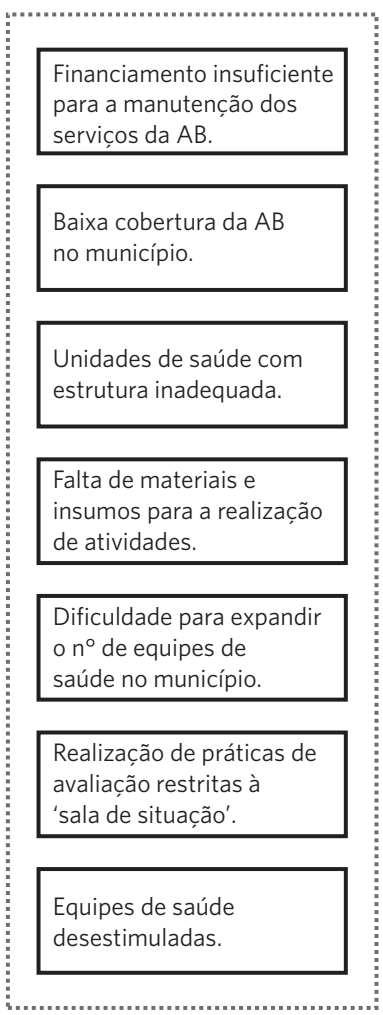

Fonte: Elaboração própria.
Objetivos

Ações

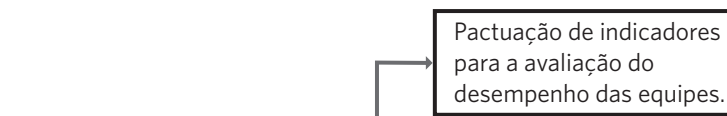

Melhorar a qualidade

da assistência

prestada pela $A B$

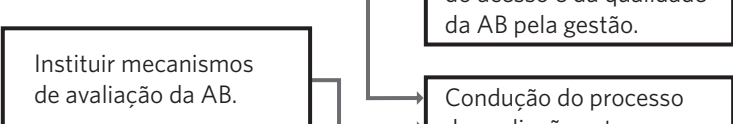

de avaliação da $A B$.

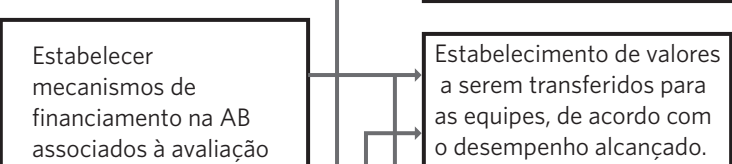

associados à avaliação

de desempenho das

equipes de saúde.

Motivar os

profissionais da $A B$.

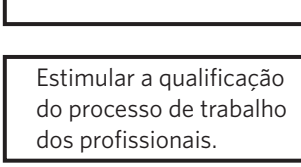

Estabelecimento de padrões para a avaliação do acesso e da qualidade de avaliação externa. Estabelecimento de valores as equipes, de acordo com o desempenho alcançado.

Definição de aplicação da porcentagem do recurso proveniente do PMAQ destinada à reestruturação da $A B$.

Melhoria dos registros das atividades desenvolvidas na $A B$.

Monitoramento de indicadores de avaliação pactuados no município.
Resultados em curto prazo
Ampliação dos recursos destinados à $A B$ municipal.

Inserção de práticas de avaliação na rotina dos profissionais e da gestão da AB.

Profissionais das unidades de saúde estimulados, como processo de avaliação proposto pelo Programa. 
O modelo lógico-operacional sintetiza e esquematiza os objetivos, as ações e os resultados do PMAQ-AB, relacionando-os com os fatores de contexto existentes no município, ou seja, o cenário de implementação do Programa ${ }^{15}$. Tais elementos se articularam com os objetivos do PMAQ-AB identificados a partir da análise das entrevistas dos gestores e profissionais, a saber: melhorar a qualidade da assistência prestada pela $\mathrm{AB}$ municipal; instituir ferramentas de avaliação nesse âmbito; estabelecer mecanismos de financiamento associados à avaliação do desempenho das equipes de saúde; motivar os profissionais; e estimular a qualificação dos processos de trabalho.

A seguir, são detalhados os principais componentes do modelo lógico-operacional do PMAQ-AB no município.

\section{Objetivos do PMAQ-AB}

Quanto ao entendimento dos objetivos do PMAQ-AB, foram observadas discordâncias entre profissionais e gestores. Ao conjecturarem sobre o assunto, os profissionais entrevistados foram unânimes em afirmar que o Programa objetivava melhorar a qualidade da assistência prestada pelas EqSF. Alguns correlacionaram o incentivo financeiro como o principal mecanismo disparador de mudanças de práticas profissionais direcionadas à melhoria da qualidade. Para outros, o PMAQ-AB objetivava melhorar as condições de trabalho na $\mathrm{AB}$.

Os gestores alegaram dificuldade de entendimento sobre os objetivos do PMAQ-AB e relataram que estavam mais voltados para mudança do processo de trabalho e para a ampliação do aporte financeiro municipal repassado pelo Ministério da Saúde (MS). Ademais, acrescentaram que as mudanças no processo de trabalho vinculavam-se aos resultados alcançados com a implantação do Programa. Esse fato demonstrou certa fragilidade na comunicação entre profissionais e gestores, e pouca concordância em relação a um aspecto importante, que é o objetivo do Programa.
Ainda que alguns objetivos tenham sido sinalizados como alcançados, poucos profissionais apontaram a existência de melhoria significativa na infraestrutura das unidades de saúde. Esse constituiu outro ponto de divergência entre profissionais e gestores, pois, ao contrário do que foi revelado pelos profissionais, os gestores afirmaram ter sido um objetivo alcançado pelo município, por meio de reformas estruturais de algumas unidades de saúde e aquisição e manutenção de equipamentos e materiais.

Outro quesito relativo ao objetivo do PMAQ-AB foi identificado no discurso dos profissionais de enfermagem ao referirem que o Programa objetivava instituir estratégias de cobrança, e que utilizava a 'avaliação' como um possível 'indutor' para um maior empenho e melhores resultados dos profissionais na AB. É provável que isto implique diretamente sobre a capacidade de as equipes identificarem a importância do PMAQ-AB, dificultando não somente a construção de planejamento, mas inclusive a fragmentação dos resultados entre as unidades de saúde da rede municipal.

Cabe destacar que essa perspectiva não foi verificada na entrevista de profissionais de outras categorias, o que pode estar relacionado à sobrecarga do profissional de enfermagem na APS, ao incluir, progressivamente, atividades administrativas, além das atividades assistenciais inerentes ao seu processo de trabalho ${ }^{22}$. Assim, vinculavam o PMAQ-AB a mais uma atribuição sob a sua condução.

\section{Identificação das ações do Programa desenvolvidas pelas equipes}

As ações desenvolvidas a partir da implantação do PMAQ-AB foram abordadas pelas equipes incluídas neste estudo. As principais ações referidas pelos profissionais foram: a melhoria nos registros e a organização das informações; o fortalecimento de práticas de promoção da saúde dentro das unidades; a pactuação de indicadores de saúde para realizar o processo de avaliação e 
monitoramento, além do acompanhamento da equipe de avaliadores e do processo de avaliação externa; e definição da aplicação dos recursos oriundos do Programa.

A melhoria nos registros e a organização das informações em saúde foram as ações mais citadas nas entrevistas, tanto pelos profissionais quanto pelos gestores participantes, que salientaram a grande contribuição do PMAQ-AB no avanço observado no processo de trabalho das equipes que aderiram ao Programa.

Atrelado a isso, nota-se o entendimento parcial de alguns profissionais a respeito de suas práticas em serviço e o processo de incorporação de novas atividades e ações, a partir da indução de mudanças de práticas, objetivada pelo PMAQ-AB. Alguns apontaram que não verificaram mudanças significativas, apenas perceberam o fortalecimento de práticas de promoção da saúde que não estavam, até então, concretizadas na rotina das equipes de saúde analisadas. Como exemplo, os entrevistados citaram: ações de educação em saúde realizadas em grupo, especialmente direcionadas à saúde dos idosos, previstas pelo Programa Nacional de Hipertensão e Diabetes Mellitus e salas de espera.

Os gestores apresentaram entendimento concordante sobre o significativo avanço na pactuação de indicadores entre os profissionais das equipes de saúde e a gestão, bem como das metas que esses indicadores deveriam alcançar. Esse processo, segundo eles, foi uma construção coletiva, que envolveu um espaço de tomada de decisão compartilhado por gestores, profissionais de saúde e apoiadores institucionais. Sobre os indicadores utilizados para a avaliação da qualidade, os profissionais consideraram alguns inadequados para avaliar a realidade local, pois apresentaram baixa coerência com as especificidades identificadas no município, a exemplo da presença de práticas integrativas, que não estão implementadas localmente. Essa percepção sobre os indicadores também foi verificada pela equipe atuante na área rural do município, que apontou a inexistência de indicadores e padrões adequados às especificidades do território rural.

No que se refere à avaliação externa, alguns profissionais de saúde apontaram como aspecto frágil o constrangimento inerente ao processo de avaliação das práticas. Tal assertiva foi explicitada pelos entrevistados, tanto de equipe da zona rural quanto urbana, ao referirem uma preocupação consensual acerca dos resultados alcançados pelas equipes. Uma ilustração sobre essa questão foi citada pelo participante E2U1 - médico, ao afirmar que

avaliar é um processo complicado, a gente se sente inseguro. Mas estávamos preparados sim, até mesmo porque fazíamos tudo que estava ao nosso alcance. Então, não foi surpresa o que pediam e perguntavam para nós.

Avaliar pressupõe emitir juízo de valor, segundo um determinado contexto histórico, social, econômico e cultural ${ }^{12}$. Desta forma, o processo avaliativo está relacionado à capacidade de reflexão crítica coletiva, especialmente, no que concerne ao processo de trabalho das equipes e à identificação de pontos que requerem melhorias, e não deve ser reduzido a uma imposição, cuja finalidade é apenas o cumprimento de metas. Mas, deve ser amplo e provocador de mudanças ${ }^{3}$.

As recomendações para a aplicação dos recursos derivados do PMAQ-AB diferem de algumas experiências internacionais. No Brasil, o repasse do incentivo financeiro é realizado fundo a fundo (do nível federal para o municipal). Contudo, os municípios possuem autonomia para estabelecer normas específicas para a utilização do recurso (por meio de adicionais pecuniários e/ou suprimentos, infraestrutura e outros estímulos que possam auxiliar a melhoria do desempenho das equipes) ${ }^{\mathbf{1 0}}$.

Outro aspecto que merece destaque é a vinculação dos critérios para a priorização de investimento ao desempenho das equipes participantes, tendo em vista que a classificação pode variar de insuficiente a muito acima da 
média. Fica a critério dos municípios optarem por investir naquelas que obtiveram maior nível de desempenho ou, alternativamente, investir nas equipes que obtiveram as pontuações mais baixas, para promovê-las. Este leque de opções pode gerar resultados não intencionais e, consequentemente, contribuir para a ampliação das desigualdades locais ${ }^{23}$.

Sobre o montante de recursos municipais, transferidos por meio do PMAQ-AB e destinados à premiação vinculada ao desempenho, segundo os entrevistados, foram geridos pelas próprias equipes, sem a intervenção da gestão municipal. Eles relataram que os profissionais de saúde que compunham as equipes definiram as estratégias para a aplicação do recurso. Nesse ponto, foi consensual que a premiação seria dividida de forma igualitária entre os integrantes da equipe.

É importante ressaltar que o processo de divisão da premiação foi permeado por opiniões divergentes. Em unidades de saúde que continham duas equipes de trabalho, os profissionais informaram que o processo de rateio gerou conflitos, em virtude das diferenças nos resultados das avaliações de cada equipe, e, consequentemente, quanto aos valores recebidos por cada.

\section{Resultados do Programa no município}

Sobre o acompanhamento dos resultados do PMAQ-AB, após os dois primeiros ciclos, os gestores apresentaram opiniões convergentes e sinalizaram aspectos favoráveis relacionados à abrangência das ações no contexto local, quais sejam: 1. A ampliação dos recursos destinados à $\mathrm{AB}$, o que permitiu melhorias na infraestrutura das unidades de saúde, e a implantação de novas unidades e equipes; 2 . A inserção de práticas de avaliação na $\mathrm{AB}$ de forma sistemática, em consonância com as diretrizes do Programa; 3. O estímulo dos profissionais da $\mathrm{AB}$, decorrente da implantação do Programa, visto que, para os gestores entrevistados, mesmo diante das dificuldades encontradas no processo de trabalho, os profissionais conseguiram resgatar a motivação e passaram a envidar maior empenho na execução das atividades.

Contudo, ainda que tenham sido observados pontos positivos na ampliação do acesso e da qualidade no município, alguns entrevistados revelaram opiniões distintas das proferidas pelos gestores. Quanto à estrutura física das unidades de saúde, todos os profissionais da área apontaram que os serviços de saúde careciam de reestruturação física para ampliarem as ações de saúde e atenderem as necessidades da população adstrita, ainda que não tenham sido consolidadas reformas estruturais abrangentes.

Do mesmo modo, os profissionais não perceberam melhoria importante no acesso após a implementação do PMAQ-AB, apesar de indicarem que a aplicação do incentivo financeiro consistiu em um mecanismo disparador de mudanças de práticas profissionais com desdobramentos, ainda que tímidos, para essa melhoria e para a qualidade da assistência prestada pela $\mathrm{AB}$. Por outro lado, os gestores não apontaram grandes mudanças com os resultados alcançados pelo Programa nos dois primeiros ciclos, o que pode constituir em um indício de que, em curto e médio prazos, essas mudanças não foram alcançadas.

Alguns autores discutem que a fragmentação da avaliação em ciclos e a alternância dos temas de intervenção, como proposto pelo PMAQ$\mathrm{AB}$, dificultam a mensuração das melhorias em longo prazo e a continuidade de projetos anteriores na vigência de novos ciclos, o que pode gerar frustração e inibir o desenvolvimento da cultura de qualidade. Esta fragmentação está relacionada à limitação de gestores e equipes para entender o planejamento, monitoramento e a avaliação como partes de um processo contínuo de trabalho por melhorias ${ }^{3}$.

\section{Análise de coerência do modelo lógico-operacional}

O modelo lógico-operacional permitiu a comparação entre o que é preconizado e o que é realizado no município, para que seja possível, 
então, compreender se as ações realizadas estão de acordo com as recomendações nacionais, conforme o modelo lógico da teoria do PMAQ-AB no âmbito nacional, proposto em um estudo de avaliaçãon ${ }^{15}$.

O PMAQ-AB, da forma que foi idealizado pelo MS, visa alcançar melhorias nos níveis de saúde da população através do desenvolvimento de processos avaliativos que levem em consideração tanto os objetivos dos serviços de saúde quanto as necessidades de saúde da população. Contudo, observou-se que, no município investigado, o modelo lógico- operacional local assume conformação menos abrangente do que o modelo teórico, que considerou as recomendações das diretrizes operacionais do Programa ${ }^{15}$.

Além disso, verificou-se uma incipiente compreensão da teoria do Programa, fato que pode ter contribuído para uma implantação parcial dos objetivos, sendo identificado no sistema local o desenvolvimento dos seguintes: melhorar a qualidade da assistência prestada pela $\mathrm{AB}$ municipal; instituir ferramentas de avaliação nesse âmbito; estabelecer mecanismos de financiamento da $\mathrm{AB}$ associados à avaliação do desempenho das equipes de saúde; motivar os profissionais da $\mathrm{AB}$; e estimular a qualificação dos processos de trabalho desses profissionais.

Desses objetivos, derivaram as ações relacionadas à pactuação de indicadores para a avaliação do desempenho das equipes; o estabelecimento de padrões para a avaliação do acesso e da qualidade da $\mathrm{AB}$ pela gestão; a condução do processo de avaliação externa; a pactuação de valores a serem transferidos para as equipes, de acordo com o desempenho alcançado; a definição de aplicação da porcentagem do recurso proveniente do PMAQ-AB destinada à reestruturação da $\mathrm{AB}$; as melhorias nos registros das atividades desenvolvidas; e o monitoramento dos indicadores pactuados para a avaliação contínua no município.

Contudo, observou-se uma priorização das ações relacionadas à obrigatoriedade do cumprimento de prerrogativas vinculadas ao componente financeiro do Programa, enquanto as ações que buscavam contemplar a melhoria da qualidade técnica e o fortalecimento das práticas de avaliação não foram evidenciadas.

Quanto aos resultados, foram identificados a ampliação dos recursos destinados à $\mathrm{AB}$ municipal e os desdobramentos dessa suplementação financeira; a inserção de práticas de avaliação na rotina dos profissionais e da própria gestão; e a melhoria no desenvolvimento das atividades dentro das unidades de saúde, em virtude do maior estímulo das equipes frente à proposta do Programa.

\section{Considerações finais}

O presente estudo alcançou o propósito de sistematizar o modelo lógico-operacional do PMAQ-AB em um município baiano. Ademais, o estudo da conformação em contextos locais permitiu analisar a pertinência do modus operandi e outros aspectos considerados relevantes das práticas de avaliação da qualidade executadas no município, para qualificá-las e torná-las mais aptas às necessidades e questões dos grupos de interesse nelas envolvidos.

A partir da análise das percepções, opiniões e do conhecimento dos profissionais e gestores entrevistados, atuantes no município investigado nos dois primeiros ciclos do PMAQ-AB, sobre o Programa, foram verificados aspectos convergentes entre algumas ponderações dos atores entrevistados, bem como a coerência do Programa desenvolvido no nível local, em comparação com a proposta governamental.

A sistematização evidenciou que, com a implementação do PMAQ-AB, em curto prazo, o município avançou em questões como: ampliação dos recursos destinados à $\mathrm{AB}$ municipal; inserção de práticas de avaliação na rotina dos profissionais e da gestão da $\mathrm{AB}$; e profissionais das unidades de saúde estimulados com o processo de avaliação proposto pelo Programa. Estes resultados estão relacionados com os pontos que deram origem à implantação do Programa, principalmente com o financiamento insuficiente para a manutenção e expansão 
dos serviços da $\mathrm{AB}$, a estrutura inadequada das unidades de saúde, a falta de materiais e insumos para a realização de atividades, as práticas de avaliação restritas à 'sala de situação’ e o desestímulo das equipes de saúde quanto ao processo de trabalho.

Do mesmo modo, a experiência prévia com processos avaliativos e a iniciativa de instituir uma cultura de avaliação na AB municipal, conforme verificado no município, pode ter facilitado o processo de compreensão do eixo 'organização de práticas avaliativas', identificado no modelo teórico nacional, e dos objetivos e ações mais voltados para esta abordagem.

Contudo, cabe destacar que a presente pesquisa se limitou a estudar os dois primeiros ciclos do Programa e, portanto, não conseguiu estabelecer os possíveis resultados em longo prazo. Outra limitação refere-se à adoção de uma análise descritiva da conformação local, a partir da percepção dos atores envolvidos, o que pode ter desdobrado em interpretações individuais que não traduziram o cenário real. Neste sentido, recomenda-se a realização de estudos que considerem o grau de implantação das ações previstas pelo Programa e permitam uma correlação com as variáveis contextuais.

Por fim, conclui-se que estudos dessa natureza contribuem para suscitar reflexões sobre a discrepância entre a intencionalidade das normas formuladas pelo âmbito federal e sua implementação, de fato, e a conformação dos programas no âmbito local, evidenciando um conjunto de indagações pertinentes para subsidiar avaliações futuras, quais sejam: a dificuldade de operacionalização decorre da falta de clareza dos pressupostos teóricos do Programa, que permitem o alinhamento entre objetivos e interesses, nos quais estão mergulhadas as intervenções em análise? Decorre da inexistência de fluxos operacionais que direcionam o processo de implementação? Ou da ausência de indicadores que viabilizem o monitoramento contínuo do alcance da implementação do Programa em contextos distintos?

\section{Colaboradoras}

Lemos LMA (0000-0001-6148-3825)* contribuiu para a concepção, o desenho, a aquisição, análise e interpretação de dados; elaborou o trabalho criticamente, para conteúdo intelectual importante; aprovou a versão final a ser publicada; e é responsável por prestar contas de todos os aspectos do trabalho, garantindo que as questões relacionadas à precisão ou integridade de qualquer parte sejam devidamente investigadas e resolvidas. Prado NMBL (0000-0001-8243-5662)* trabalhou para a concepção, delineamento e processamento dos dados, avaliação da versão final; e é responsável por prestar conta de todos os aspectos do trabalho, garantindo que as questões relacionadas à precisão ou integridade de qualquer parte sejam devidamente investigadas e resolvidas. Medina MG (0000-0001-7283-2947) contribuiu para a concepção do trabalho e análise de dados; revisou criticamente o conteúdo intelectual importante; aprovou versão final a ser publicada; e é responsável por prestar contas de todos os aspectos do trabalho, garantindo que as questões relacionadas à precisão ou integridade de qualquer parte sejam devidamente investigadas e resolvidas. 


\section{Referências}

1. Fertonani HP, Pires DEP, Biff B, et al. Modelo assistencial em saúde: conceitos e desafios para a atenção básica brasileira. Ciênc. Saúde Colet. 2015; 20(6):18691878.

2. Giovanella L, Mendonça MHM. Atenção Primária à Saúde. In: Giovanella L, Escorel S, Lobato LVC, et al., organizadores. Políticas e Sistema de Saúde no Brasil. Rio de Janeiro: Fiocruz; 2012.

3. Silva RR, Furtado JP, Akerman M, et al. Subsídios à meta-avaliações do PMAQ. In: Akerman M, Furtado JP. Práticas de avaliação em saúde no Brasil - diálogos. Porto Alegre: Reunida; 2016. p. 327-355.

4. Clementino FS, Gomes LB, Vianna RPT, et al. Acolhimento na Atenção Básica: análise a partir da avaliação externa do Programa de Melhoria do Acesso e da Qualidade da Atenção Básica (PMAQ-AB). Rev. Saúde Ciênc. Online. 2015; 4(1):62-80.

5. Brasil. Ministério da Saúde, Secretaria de Atenção à Saúde, Departamento de Atenção Básica. Programa Nacional de Melhoria do Acesso e da Qualidade da Atenção Básica (PMAQ-AB): manual instrutivo. Brasília, DF: Ministério da Saúde; 2012.

6. Cruz MM, Souza RBC, Torres RMC, et al. Usos do planejamento e autoavaliação nos processos de trabalho das equipes de Saúde da Família na Atenção Básica. Saúde debate. 2014; 38(esp):124-139.

7. Fausto MCR, Giovanella L, Mendonça MHM, et al. A posição da Estratégia Saúde da Família na rede de atenção à saúde na perspectiva das equipes e usuários participantes do PMAQ-AB. Saúde debate. 2014; 38(esp):13-33.

8. Belo FMA, Carvalho EF, Farias SF. Análise do Programa de Melhoria do Acesso e da Qualidade da Atenção Básica em Recife-PE. Saúde debate. 2014; 38(103):706719.

9. Sossai TA, Galavote HS, Vieira ECL, et al. Evidências sobre o Programa Nacional de Melhoria do Acesso e da Qualidade da Atenção Básica. Rev. Bras. Pesq. Saúde. 2016; 18(1):111-119.

10. Moraes PN, Iguti AM, Avaliação do desempenho do trabalhador como forma peculiar de prescrição do trabalho: uma análise do PMAQ-AB. Saúde debate. 2013; 37(98):416-426.

11. Lopes EAA, Scherer MDA, Costa AM. O Programa Nacional de Melhoria do Acesso e da Qualidade da Atenção Básica e a organização dos processos de trabalho. Tempus, Actas de Saúde Colet. 2015; 9(2):237250.

12. Hartz ZMA, Vieira-da-Silva LM. Avaliação em saúde: dos modelos teóricos à prática na avaliação de programas e sistemas de saúde. Rio de Janeiro: Fiocruz; 2005.

13. Champagne F, Brouselle A, Hartz Z, et al. Modelizar as intervenções. In: Brousselle A, Champagne F, Contandriopoulos AP, et al., organizadores. Avaliação: Conceitos e métodos. Rio de Janeiro: Fiocruz; 2011. p. 61-74.

14. Brasil. Ministério da Saúde. Datasus. Informações de Saúde. Indicadores de Saúde e Pactuações. 2015. [internet] [acesso em 2018 jul 1]. Disponível em: http:// tabnet.datasus.gov.br/cgi/tabcgi.exe?pacto/2015/cnv/ coapmunba.def.

15. Lemos LMA, Prado NMBL, Medina MG. Programa Nacional de Melhoria do Acesso e da Qualidade da Atenção Básica (PMAQ-AB): modelização da política no âmbito nacional. Soc. e Cult. 2018; 21(2):54-72.

16. Brousselle A, Champagne F, Contandriopoulos AP, et al., organizadores. Avaliação: conceitos e métodos. Rio de Janeiro: Fiocruz; 2011.

17. Medeiros KRG, Junqueira AGW, Schwingel G, et al. A rotatividade de enfermeiros e médicos: um impasse na implementação da Estratégia de Saúde da Família. Ciênc. Saúde Colet. 2014; 15(1):1521-1531. 
18. Albuquerque IMN, Santos LTV, Dias FIS, et al. Sala de situação para tomada de decisão: percepção dos profissionais que atuam na atenção básica à saúde de Sobral - Ceará. Sanare, 2013; 12(2):40-46.

19. Vitória da Conquista. Lei ${ }^{0} 1.907$, de 06 de junho de 2013. Dispõe sobre o pagamento do Prêmio Variável de Qualidade e Inovação, do Programa Nacional de Melhoria do Acesso e da Qualidade da Atenção Básica e do incentivo financeiro (PMAQ-AB), denominado componente de qualidade do piso de atenção básica, instituído pelo Ministério da Saúde, e dá outras providências. Vitória da Conquista, 06 de junho de 2013. [acesso em 2020 maio 20]. Disponível em: http://ba.portaldatransparencia.com.br/prefeitura/ vitoriadaconquista/index.cfm?pagina=abreDocume nto\&arquivo=32E3025C8146.

20. Vitória da Conquista. Prefeitura Municipal. Rede Básica de Saúde Vitória da Conquista participa do Programa Nacional de Melhoria do Acesso e da Qualidade. [internet]. $27 \mathrm{dez}$ 2013. [acesso em 2020 jun 11]. Disponível em: http://www.pmvc.ba.gov.br/rede-basica-de-saude-vitoria-da-conquista-participa-do-programa-nacional-de-melhoria-do-acesso-e-da-qualidade-da-atencao-basica/.
21. Pinto HA, Sousa ANA, Ferla AA. O Programa Nacional de Melhoria do Acesso e da Qualidade da Atenção Básica: várias faces de uma política inovadora. Saúde debate. 2014; 38(esp):358-372.

22. Rizzotto MLF, Gil CRR, Carvalho M, et al. Força de trabalho e gestão do trabalho em saúde: revelações da Avaliação Externa do Programa Nacional de Melhoria do Acesso e da Qualidade da Atenção Básica no Paraná. Saúde debate. 2014; 38(esp):237-251.

23. Macinko J, Harris MJ, Rocha MG. Brazil's National Program for Improving Primary Care Access and Quality (PMAQ) Fulfilling the Potential of the World's Largest Payment for Performance System in Primary Care. J Ambulatory Care Manage. 2017; 40(2):411.

Recebido em 02/06/2019

Aprovado em 07/05/2020

Conflito de interesses: inexistente

Suporte financeiro: não houve 